\title{
BMJ Open Cohort profile: the MUNICH Preterm and Term Clinical study (MUNICH-PreTCl), a neonatal birth cohort with focus on prenatal and postnatal determinants of infant and childhood morbidity
}

\author{
Susanne Pangratz-Fuehrer (D) , ${ }^{1}$ Orsolya Genzel-Boroviczény, ${ }^{1}$ \\ Wolfgang Bodensohn (D) , ${ }^{1}$ Robin Eisenburger, ${ }^{1}$ Janne Scharpenack, ${ }^{1}$ \\ Philipp E Geyer, ${ }^{2,3}$ Johannes B Müller-Reif, ${ }^{2,3}$ Nadja van Hagen, ${ }^{1}$ Alina M Müller, ${ }^{1}$ \\ Majken Karoline Jensen, ${ }^{4,5}$ Christoph Klein, ${ }^{6}$ Matthias Mann, ${ }^{2,7}$ Claudia Nussbaum ${ }^{1}$
}

To cite: Pangratz-Fuehrer S, Genzel-Boroviczény 0, Bodensohn W, et al. Cohort profile: the MUNICH Preterm and Term Clinical study (MUNICH-PreTCI), a neonatal birth cohort with focus on prenatal and postnatal determinants of infant and childhood morbidity. BMJ Open 2021;11:e050652. doi:10.1136/ bmjopen-2021-050652

- Prepublication history for this paper is available online. To view these files, please visit the journal online (http://dx.doi. org/10.1136/bmjopen-2021050652).

Received 01 March 2021 Accepted 20 May 2021

D Check for updates

(c) Author(s) (or their employer(s)) 2021. Re-use permitted under CC BY-NC. No commercial re-use. See rights and permissions. Published by BMJ.

For numbered affiliations see end of article.

\section{Correspondence to}

Dr Susanne Pangratz-Fuehrer; Susanne.Pangratzfuehrer@med. uni-muenchen.de

\section{ABSTRACT}

Purpose The MUNICH Preterm and Term Clinical (MUNICHPreTCI) birth cohort was established to uncover pathological processes contributing to infant/childhood morbidity and mortality. We collected comprehensive medical information of healthy and sick newborns and their families, together with infant blood samples for proteomic analysis. MUNICHPreTCl aims to identify mechanism-based biomarkers in infant health and disease to deliver more precise diagnostic and predictive information for disease prevention. We particularly focused on risk factors for pregnancy complications, family history of genetically influenced health conditions such as diabetes and paediatric long-term health—all to be further monitored and correlated with proteomics data in the future.

Participants Newborns and their parents were recruited from the Perinatal Center at the LMU University Hospital, Munich, between February 2017 and June 2019. Infants without congenital anomalies, delivered at 23-41 weeks of gestation, were eligible.

Findings Findings to date concern the clinical data and extensive personal patient information. A total of 662 infants were recruited, $44 \%$ were female ( $36 \%$ in preterm, $46 \%$ in term). $90 \%$ of approached families agreed to participate. Neonates were grouped according to gestational age: extremely preterm ( $<28$ weeks, $\mathrm{N}=28$ ), very preterm ( 28 to $<32$ weeks, $\mathrm{N}=36$ ), late preterm (32 to $<37$ weeks, $\mathrm{N}=97$ ) and term infants ( $>37+0$ weeks, $\mathrm{N}=501)$. We collected over 450 data points per child-parent set, (family history, demographics, pregnancy, birth and daily follow-ups throughout hospitalisation) and 841 blood samples longitudinally. The completion rates for medical examinations and blood samples were $100 \%$ and $95 \%$ for the questionnaire.

Future plans The correlation of large clinical datasets with proteomic phenotypes, together with the use of medical registries, will enable future investigations aiming to decipher mechanisms of disorders in a systems biology perspective.

Trial registration number DRKS (00024189); Pre-results.

\section{Strengths and limitations of this study}

- The MUNICH Preterm and Term Clinical study is a prospective birth cohort consisting of 662 infants (501 full term and 161 preterm) recruited from a single Perinatal Center in Germany.

- We collected phenotypical information including clinical data from maternal and neonatal medical records, family history and demographics survey and blood samples at defined time points for proteomic screening.

- Recruiting at the university hospital and restricting the survey to German and English-speaking parents likely introduced some bias as mostly caucasian families from urban living environment and with a higher educational level participated in the study.

- Combining neonatal cohort studies with proteomic screening provides an opportunity to relate the functional protein network status to specific prenatal and postnatal factors as well as clinical outcomes recorded at time of birth and during follow-up.

- This hypothesis-free approach may enable the identification of biomarkers for the aetiologic understanding of complex multifactorial short and longterm diseases in infants.

\section{INTRODUCTION}

Early life exposure to environmental impact factors (eg, air pollution, noise, chemicals and pesticides) and family medical history can alter ontogenic trajectories in fundamental and unforeseen ways. These often result in clinically important outcomes during pregnancy, at birth and in the long term. In particular, preterm birth is still associated with an increased incidence of complications, despite advances in neonatal intensive care. ${ }^{1}$ The pathogenesis is thought to be of multifactorial origin, involving the exacerbating 
interaction of genetic components with a multitude of environmental risk factors. ${ }^{2}$ Furthermore, it is conceivable, that poor outcomes among preterm infants are not solely associated with being born too early, but that the underlying reasons for prematurity itself could be of even greater importance. ${ }^{3}$

This raises the question why some infants develop diseases, while others are resilient despite potentially carrying a heightened risk for morbidity. Resilient individuals may provide important clues for improved disease prevention but can only be identified when patients are compared within a particular group sharing a defining characteristic or common event, such as birth. This highlights the need for population-based pregnancy groups and birth cohorts to investigate environmental and genetic factors with the purpose of increasing our understanding of the origins of health and disease-starting as early as in prepregnancy. Previous neonatal cohort studies have used maternal biomarkers to explore the influence of potentially disease-causing environmental factors on long-term health outcomes of the child. ${ }^{45}$ Moreover, prepregnancy obesity and abnormal weight gain during early gestation have been associated with an adverse cardiometabolic profile in the offspring, including, but not limited to higher childhood body mass index (BMI) and systolic blood pressure. ${ }^{6}$ In particular, the third trimester-a critical period for brain and lung development as well as metabolic programming - is now understood to be remarkably sensitive to disruptive factors like alcohol and drug abuse, stress and malnutrition. ${ }^{7}$ The advantage of cohort studies combining clinical data, family history and the collection of body fluids during hospitalisation is the opportunity for an unbiased assessment of circulating proteins in blood and plasma via highly informative 'OMICS' technologies including genomics, microbiomics and plasma proteome profiling enable in-depth phenotyping of the individual. ${ }^{8}$ The results of biospecimen measurements can then be related to specified prenatal and postnatal factors as well as clinical outcomes recorded at time of birth and during follow-up. This hypothesis-free approach may enable the identification of biomarkers and disease-modifying effects for the aetiologic understanding of complex multifactorial short and long-term diseases in infants.

\section{COHORT DESCRIPTION}

The MUNICH Preterm and Term Clinical study (MUNICH-PreTCl) is a prospective cohort of 662 neonates. Between February 2017 and June 2019, we included 501 full-term neonates recruited from our maternity ward and 161 preterm infants on admission to our neonatal intensive care unit at the Perinatal Center, Campus Innenstadt, University Hospital, LMU, Munich, Germany. For preterm infants, we defined three subgroups as follows: infants born at less than 28 completed weeks of gestation (extremely preterm, $\mathrm{N}=28$ ), infants born between
$28+0$ and $31+6$ weeks (very preterm, $\mathrm{N}=36$ ) and infants born between $32+0$ and $36+6$ weeks (late preterm, $\mathrm{N}=97$ ).

\section{Study aims}

MUNICH-PreTCl was designed as a comprehensive neonatal cohort study, enrolling both, preterm and term infants. Our overall objectives were to first elucidate the role of gestational age (GA), prenatal and postnatal environmental exposures and family demographic and medical history in determining the risk of neonatal morbidity among preterm and term infants. Second, we wanted to establish a thorough baseline assessment for future follow-ups regarding disease occurrence. To this end, we systematically collected comprehensive datasets on healthy infants with and without specific susceptibilities to diseases, on sick infants and on critically ill infants. We monitored the medical status of enrolled neonates, recorded their families' backgrounds and obtained blood samples for proteomic analysis from each infant. With these data at hand, we can investigate the impact of specific medical and environmental conditions on neonatal health outcomes in an attempt to preventively improve the lives of preterm infants, critically ill patients and their families.

\section{Study population}

Participant recruitment and informed consent

Mothers of eligible infants were approached for enrolment after giving birth at the Perinatal Center, Campus Innenstadt, University Hospital, LMU, Munich, Germany. Full informed consent was given by mothers for the formation of a comprehensive data set derived from maternal and infant medical records, a parental questionnaire and for collecting blood samples from the infant during routine blood sampling. MUNICH-PreTCl was approved by the ethics board of the Medical Faculty of the LudwigMaximilians-University, Munich, Germany.

Our patient recruitment process consisted of three stages, with stage 1 and 3 being specifically dedicated to preterm infants. During stage 1 (pilot phase), we enrolled neonates with a GA of 23-36 completed weeks, born between February 2017 and May 2018, aiming to establish a workflow for patient recruitment and sample collection for proteomic analyses. We collected data throughout the hospitalisation of mothers and infants including their medication and medical procedures, but we did not record their detailed family history and demographics.

Stage 2 commenced in June 2018 and continued through December 2018. During this stage, we expanded the recruitment focus by enrolling preterm and term infants as well as their parents, who received a comprehensive health survey with detailed medical and family history to determine the role of potentially modifiable factors contributing to long-term developmental outcome.

Stage 3 was launched in January 2019 essentially constituting an extension of stage 2 but with the objective of collecting a maximum number of extremely preterm and very preterm infants as well as augmenting specific 
groups of interest, such as neonates with neonatal infections, diabetic mothers or being 'small for GA (SGA)' (< 10th age-adjusted weight percentile).

\section{Clinical data and questionnaire}

Data were collected from enrolled infants throughout their hospital stay using medical records, results from medical and laboratory examinations and parental questionnaires to characterise current and previous pregnancies and births, education, life style patterns as well as chronic health outcomes of their families. Events before or during pregnancy were documented retrospectively. The questionnaire was handed out to the parents of 582 infants and completed by 550 (95\% participation rate). Since the survey did not start until stage 2 , a subsequently smaller number of preterm infants' parents submitted the family questionnaire (78 out of 90 preterm infants and 472 out of 492 term infants). Furthermore, the survey participation rate for families with extremely preterm infants was only $55 \%$ and thus much lower compared with the other preterm age groups with $77 \%$ for very preterm and $94 \%$ for late preterm infants.

GA was estimated by experienced obstetricians using the mother's last menstrual period as well as the first trimester ultrasound. GA is expressed in completed weeks (or completed days), such that events occurring 210-216 completed days after the onset of the last period were considered to be at 30 weeks of gestation. Data were then entered onsite into a secure and pseudonymised database by trained doctoral students with password protection for confidentiality.

\section{Blood samples}

Infant blood samples were taken at predefined time points: at first routine blood sampling after birth, at newborn screening 36-48hours after birth, preantibiotic and postantibiotic treatment, at adjusted 32 weeks of GA and at discharge from the clinic. Blood spots were collected on Whatman cards, which were stored frozen at $-80^{\circ}$ for mass spectrometry-based proteomic analysis. Table 1 provides an overview of domains and measurements collected in the course of MUNICH-PreTCl.
We identified two main reasons for failure to enrol eligible patients in MUNICH-PreTCl:

First, the language barrier-insufficient German or English language proficiency and second, organisational and logistical challenges due to parents' absence. Notably, some parents enrolled their newborn in our study but declined to complete the questionnaire. This was rarely observed for families of term infants $(5 \%)$, but mainly for parents of extremely (45\%) and very preterm infants (23\%), who appeared to be exhausted having to deal with long-term hospitalisation and the illness of their premature child. During stage 1, 94 families out of 294 eligible infants were approached by our study team and 80 of these subsequently enrolled their child, while parents of 14 infants declined recruitment (85\% participation). For stage 2, we identified 1173 eligible patients of preterm and term infants. Parents of 619 of these eligible patients were approached and 549 consented to study participation $(89 \%)$. For 520 of them, a completed survey could be secured (95\% participation). In stage 3 , we contacted the families of 38 of 265 eligible patients, of which 33 were enrolled and 5 declined consent (87\% participation). Figure 1 provides the study flowchart for the selection and enrolment process.

\section{Baseline characteristics of neonatal study cohort}

The baseline characteristics of all enrolled infants are presented in table 2. Overall, a smaller proportion of participants in this cohort is female $(44 \%)$, in particular, within the group of preterm infants $(36 \%)$ compared with the term group (46\%). These numbers are in accordance with previous studies that report preterm birth to be more common $(55 \%)$ in male infants. ${ }^{9}$ Not only the GA but also the birth weight is pivotal in the classification of an infant's condition. In our cohort, the mean birth weight was $3422 \mathrm{~g}$ for term infants, $725 \mathrm{~g}$ for extremely preterm, $1299 \mathrm{~g}$ for very preterm infants and $2240 \mathrm{~g}$ for late preterm infants. As per the WHO, the term 'low birth weight' (LBW) is defined as an absolute weight of $<2500 \mathrm{~g}$, regardless of GA, and can be further categorised into very LBW (VLBW $<1500 \mathrm{~g}$ ) and extremely LBW

Table 1 Overview of domains and measurements for MUNICH-PreTCI

\begin{tabular}{|c|c|c|c|c|c|}
\hline Domains & Child & Parents & Siblings & $\begin{array}{l}\text { Extensive } \\
\text { family }\end{array}$ & Assessment \\
\hline $\begin{array}{l}\text { Infants: birth characteristics, measurements, } \\
\text { medical complications, treatments (table 2) }\end{array}$ & $x$ & & & & Medical records \\
\hline $\begin{array}{l}\text { Pregnancy: prenatal screenings, influences on } \\
\text { pregnancy, substance abuse (table } 3 \text { ) }\end{array}$ & & $x$ & $x$ & & $\begin{array}{l}\text { Medical records; self- } \\
\text { report }\end{array}$ \\
\hline $\begin{array}{l}\text { Delivery : duration, anaesthesia, previous } \\
\text { deliveries, abortions (table 4) }\end{array}$ & & $x$ & $x$ & & $\begin{array}{l}\text { Medical records; self- } \\
\text { report }\end{array}$ \\
\hline $\begin{array}{l}\text { Family medical history: allergies, } \\
\text { cardiovascular, endocrine, neurological } \\
\text { disorders (table 6) }\end{array}$ & & $x$ & $x$ & $x$ & $\begin{array}{l}\text { Medical records; self- } \\
\text { report }\end{array}$ \\
\hline
\end{tabular}


Infants, born at 23-41 weeks of gestation,

between 02/2017 - 06/2019, Perinatal Center, Innenstadt, LMU University Hospital in Munich, $\mathbf{N}=\mathbf{5 6 0 3}$

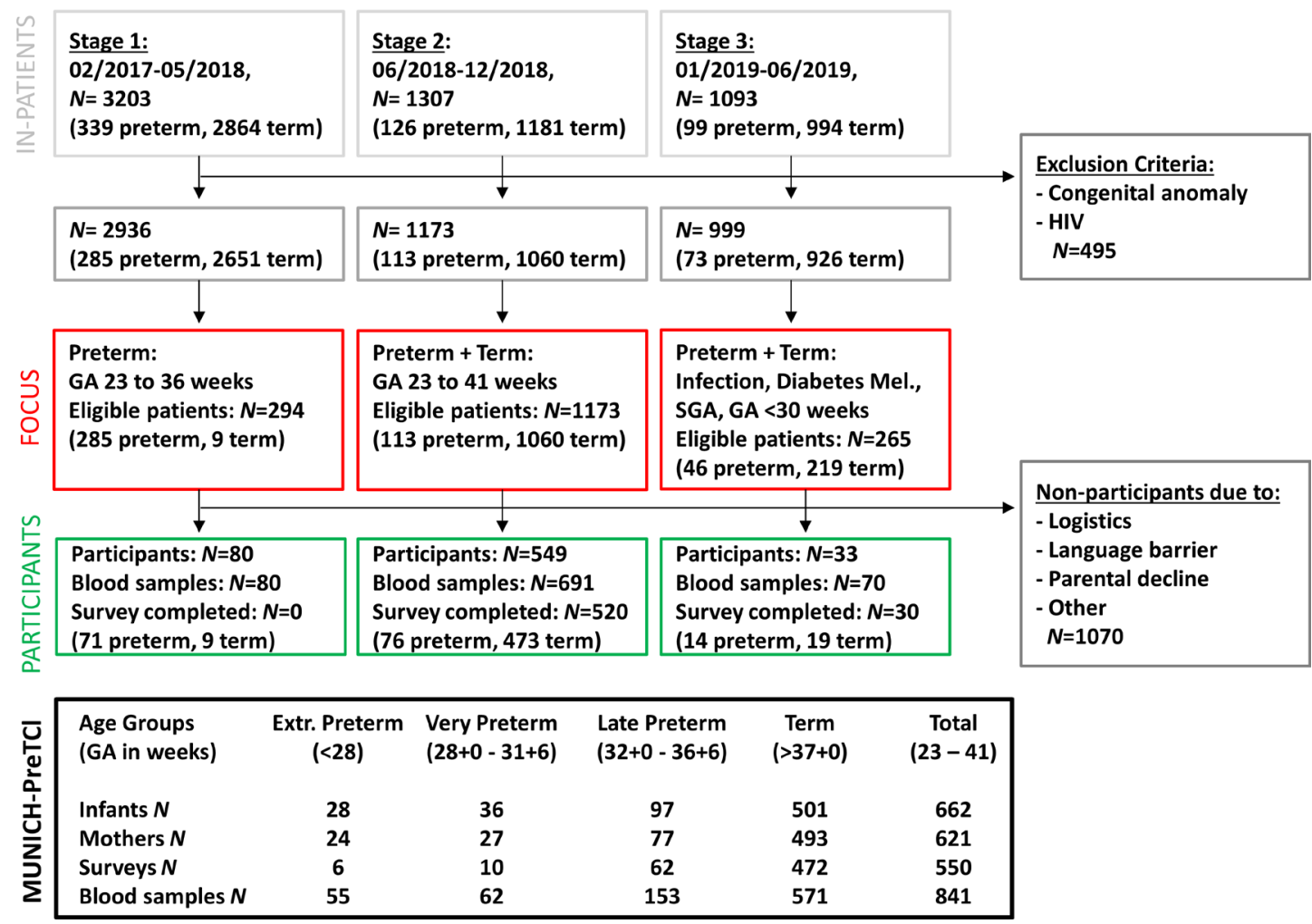

Figure 1 Study flowchart. GA, gestational age; SGA, small for GA.

(ELBW $<1000 \mathrm{~g}$ ), which generally comprises the youngest preterm infants with highest risk for complications. Among all preterm infants, $33(21 \%)$ can be categorised as ELBW and $62(39 \%)$ as VLBW. LBW can also be an indicator for the infant being too 'SGA', which refers to infants whose birth weight is below the 10th percentile for GA, due to slow prenatal growth rates caused by maternal health issues, placental complications or genetics. ${ }^{10}$ Neonates born 'large for GA' (LGA), defined as weight above the 90th percentile, are also associated with significantly higher rates of neonatal morbidity. ${ }^{11}$ There was a higher proportion of SGA infants in the group of extremely preterm infants $(21 \%)$ compared with any other group $(6 \%-12 \%)$, while the distribution of LGA infants was about equal for each GA (3\%-5\%). Infants born at the earliest GA are at the highest risk for severe morbidities and adverse outcome. As expected, postnatal complications were much more frequent in the preterm group and decreased with each advancing week of gestation, which is reflected in the following data: The incidence of cardiovascular conditions typically associated with prematurity, including arterial hypotension and hypertension, atrial septal defects, patent foramen ovale and patent ductus arteriosus, was highest in the youngest preterm infants. The percentage of infants with haematological diagnoses (anaemia of prematurity, polycythemia, thrombocytopenia, coagulation disorders) was $79 \%$ for extremely preterm and $4 \%$ for term infants.
Furthermore, as expected, the prevalence of infections was much higher in the extremely preterm infant group compared with the term infant group ( $75 \%$ for extremely preterm; $8 \%$ for term). For neurological abnormalities, no infant developed periventricular leukomalacia. Nonetheless, findings such as intraventricular and intracerebral haemorrhage, hypoxic ischaemic encephalopathy, periventricular echodensities and increased latencies of auditory brainstem responses, the highest percentage was $41 \%$ for extremely and very preterm infants, compared with $3 \%$ for term infants.

Respiratory complications, such as respiratory distress syndrome caused by primary or secondary surfactant deficiency, respiratory failure, defined as secondary worsening of lung function with hypoxemia/hypercapnia requiring exacerbation of ventilatory support, apneoa, bronchopulmonary dysplasia and pneumothorax, were most prevalent in patients $<32$ weeks of GA $(89 \%-100 \%)$ compared with term infants (3\%). Due to our focus on recruiting early preterm infants and infants with infections during stage 3 of our study, the high percentage of neonates at less than 32 weeks of gestation who had received antibiotic treatment $(95 \%)$ was predictable. As could be expected, the majority of extremely and very preterm infants required surfactant treatment (79\%) in addition to ventilatory support $(100 \%$ for extremely and $97 \%$ for very preterm), only $45 \%$ of late preterm infants and $8 \%$ of term infants needed ventilation. Data on the 
Table 2 Baseline characteristics of study participants

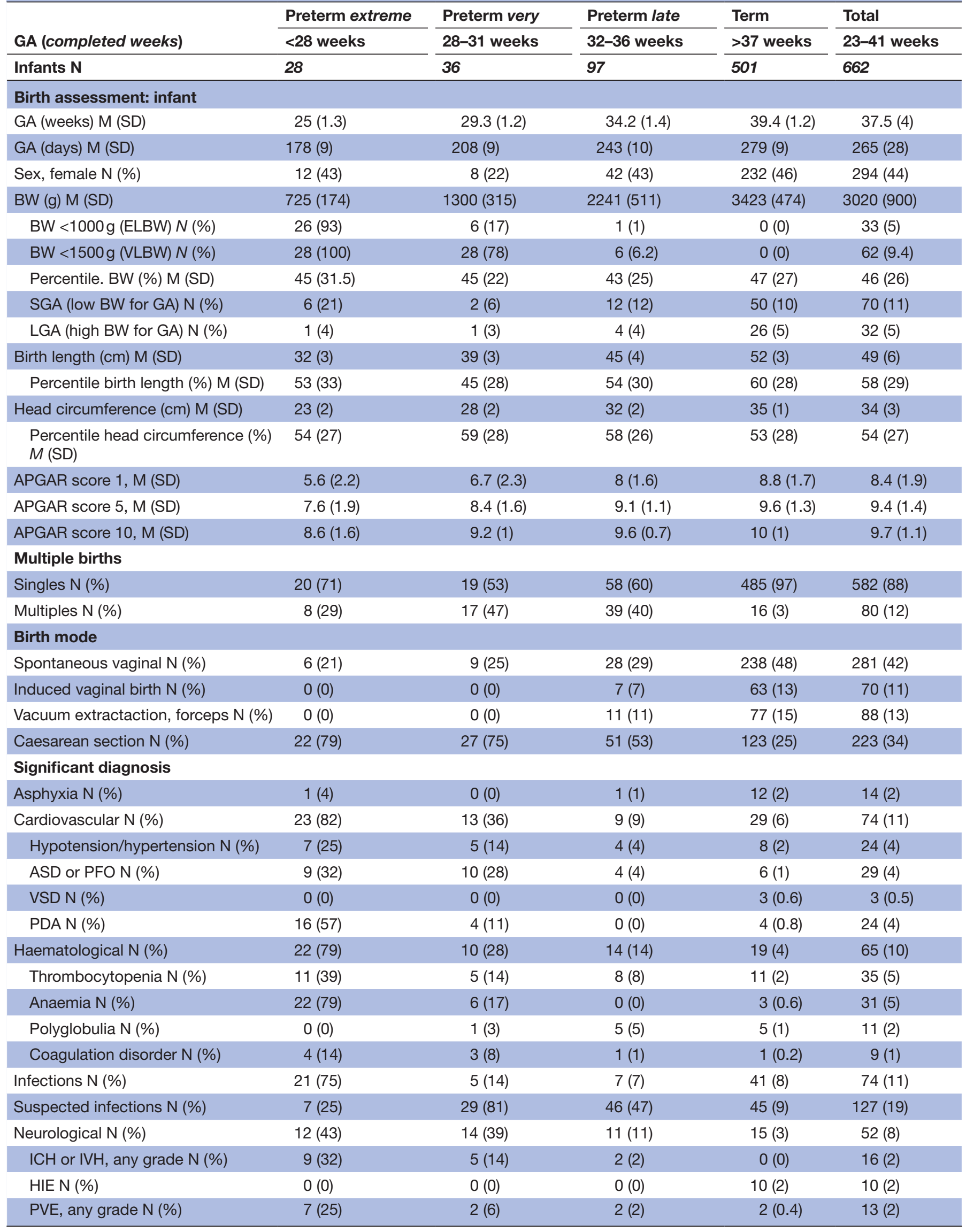


Table 2 Continued

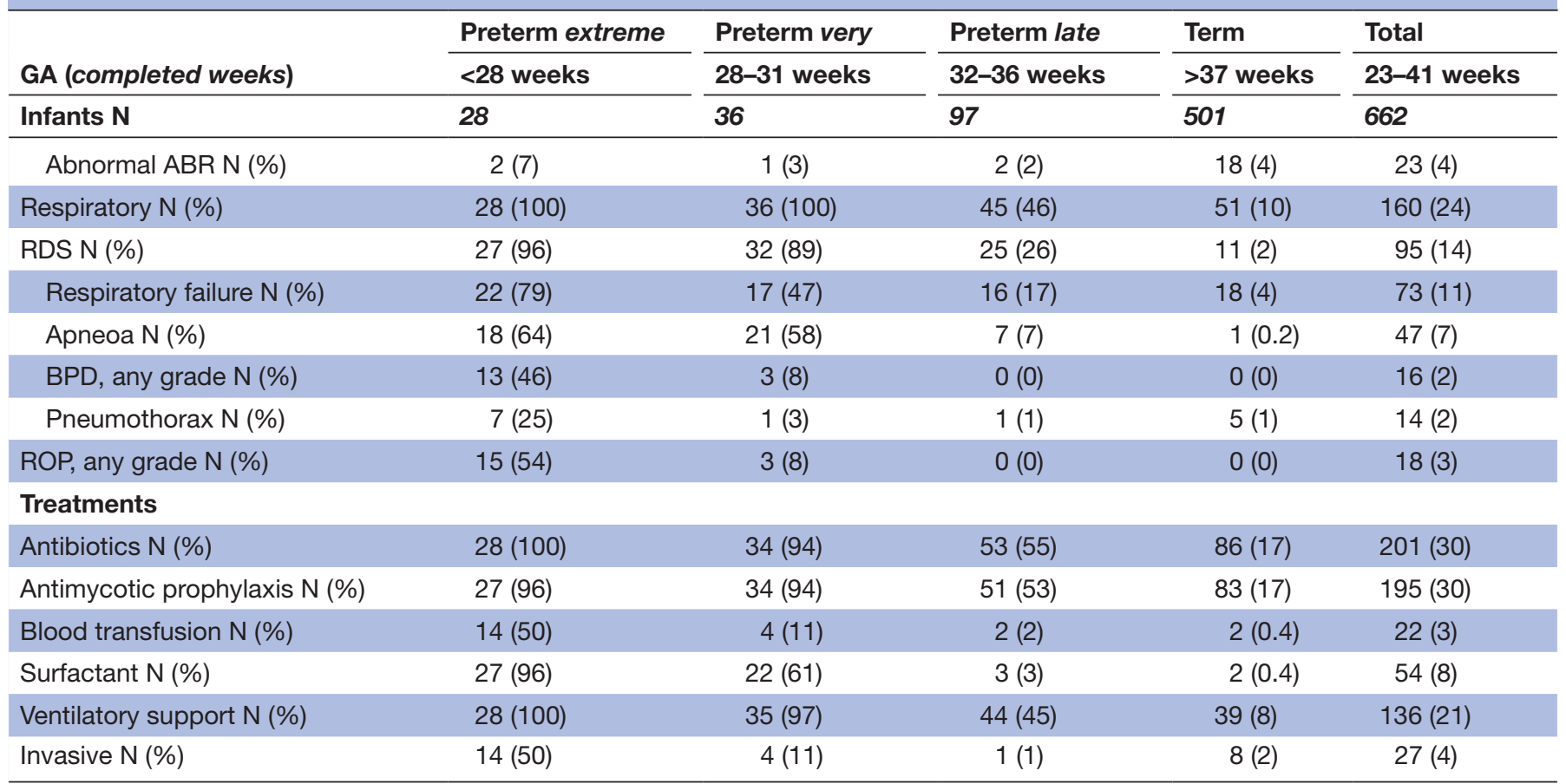

ABR, auditory brainstem response; APGAR, appearance, pulse, grimace, activity, and respiration; ASD, atrial septal defect; BPD, bronchopulmonary dysplasia; BW, birth weight; ELBW, extremely low birth weight; GA, gestational age; HIE, hypoxic ischaemic encephalopathy; ICH, intracerebral haemorrhage; IVH, intracerebral haemorrhage; LGA, large for GA; PDA, patent ductus arteriosus; PFO, patent foramen ovale; PVE, periventricula echodensities; RDS, respiratory distress syndrome; ROP, retinopathy of prematurity; SGA, small for GA; VLBW, very low birth weight; VSD, ventricular septal defect.

primary mode of respiratory support, such as invasive (INV) versus non-INV ventilation, as well as their respective subtypes, including intermittent positive pressure ventilation, continuous positive airwave pressure and high-flow nasal cannula, were collected.

\section{Baseline characteristics of prenatal care and pregnancy}

Table 3 provides an overview of prenatal care and potential influences on pregnancy. The data shown were obtained from clinical records and additional information was collected through the questionnaire (marked with * in the table). Prenatal care in Germany starts at 10-12 weeks of gestation and consists of 12 regular check-up appointments, one every 4 weeks until week 32, and every 2 weeks thereafter. Among all mothers, more than $80 \%$ received their first check-up within the initial 10 weeks of pregnancy. During routine prenatal screenings, gestational diabetes mellitus (GDM) was detected in $6 \%$. As the test for GDM is routinely performed between 24 and 28 weeks of gestation, the majority of mothers of extremely preterm infants $(71 \%)$ delivered their child before the test was performed. Only a small percentage of mothers (14\%) underwent testing for chromosomal abnormalities. Due to specific risk factors, such as advanced maternal age ( $47 \% \geq 35$ years), nicotine abuse or individual maternal health problems, nearly $70 \%$ of pregnancies were defined as 'risk pregnancies'. Within this group, $76 \%$ were mothers of preterm and $67 \%$ of term infants. The majority of mothers $(>95 \%)$ was tested for
'TORCH'-infections (toxoplasmosis, others, rubella, cytomegalovirus, herpes), which could be passed onto their fetuses during pregnancy (data not shown). Another risk factor for pregnancy complications is overweight. Obesity and excessive gestational weight gain (GWG) are associated with increased risk for GDM and gestational hypertension (GHT), preeclampsia, delivery of LGA infants and a higher incidence of congenital defects. ${ }^{12}$

Guidelines for pregnant women are recommending a BMI of 18.5-24.9 and GWG of 11.5-16 kg. ${ }^{13}$ The women's prenatal BMI shown in table 3 was recorded during their first prenatal check-up (usually between 10 and 12 weeks of the pregnancy). In comparison with the prepregnancy BMI (shown in the Baseline characteristics of parental study participants), there were only minor changes. The GWG calculated for the entire length of the pregnancy was for the term group $14 \mathrm{~kg}$, which is within the limits for women with a healthy BMI. Multiplicity is another strong risk factor for preterm birth and postnatal complications. ${ }^{14}$ Among all mothers of this study, there was a clear group difference for multiplicity: for term infants, $2 \%$ (9 out of 493) of mothers were pregnant with multiples, compared with $26 \%$ (33 out of 128) within the preterm group and 13 of these 33 mothers (40\%) had conceived via assisted reproductive technology. This is in particular interesting, as a growing body of evidence describes an increased risk of cerebral palsy in children conceived by assisted reproduction, which is strongly 
Table 3 Baseline characteristics of prenatal care and pregnancy

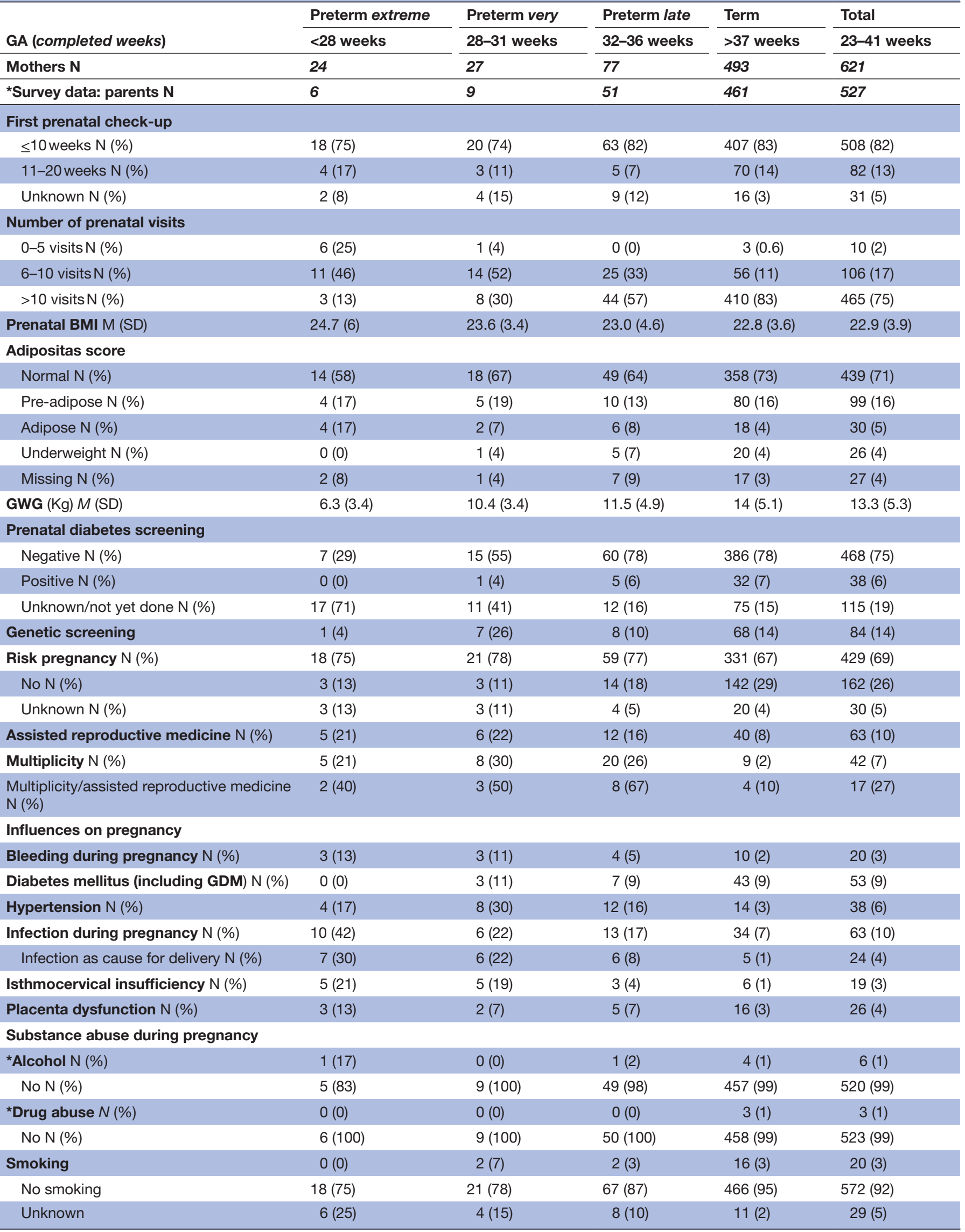


Table 3 Continued

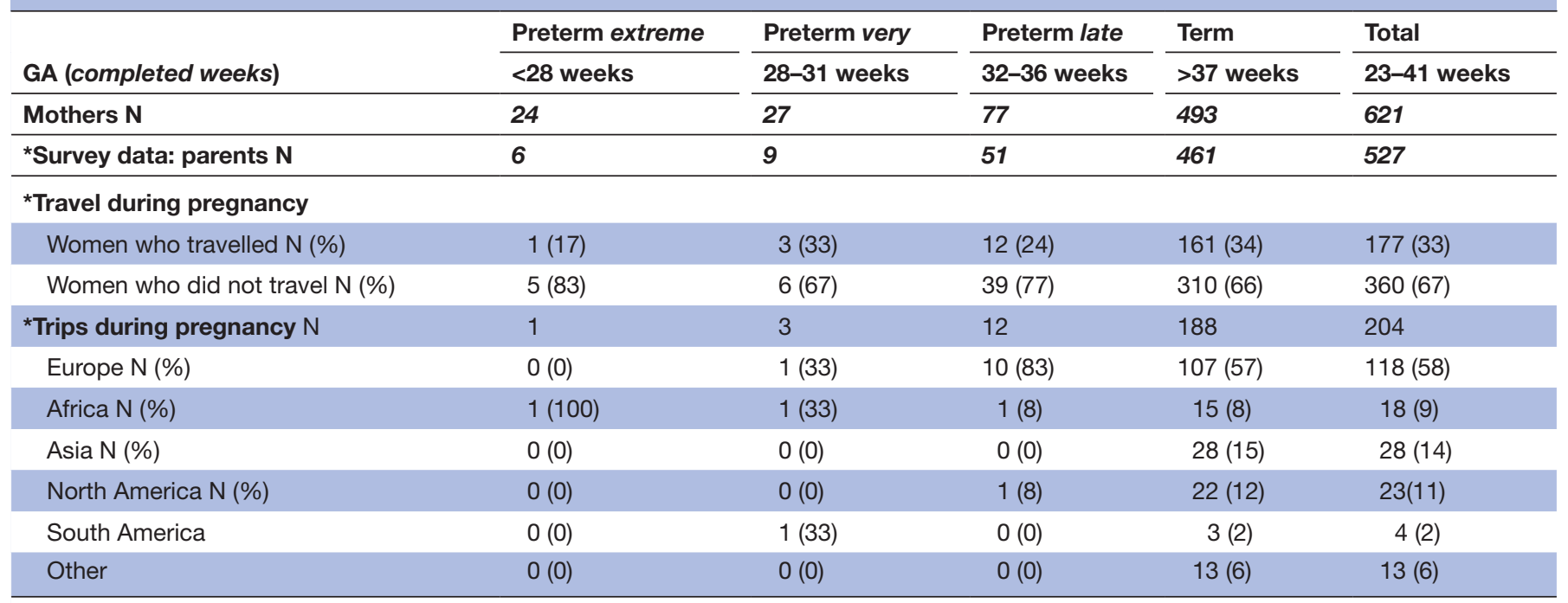

BMI, body mass index; GA, gestational age; GDM, gestational diabetes mellitus; GWG, gestational weight gain.

associated with the high proportion of multiplicity and preterm delivery in these pregnancies. ${ }^{15} 16$ Furthermore, we screened for well-researched associations of GA and determinants for preterm birth. While infections play a key role in the pathogenesis of prematurity, it is necessary to distinguish between intrauterine infections, such as the amniotic infection syndrome (AIS), mostly resulting in preterm delivery, and other types of maternal infections, including influenza, lyme disease or herpes virus infection. As expected, there was a higher prevalence for maternal infections in the preterm group, where 21 out of 29 affected women (72\%) had AIS (not shown). For $19(65 \%)$ of these mothers, this led to induced preterm delivery. For the term group, a total of $7 \%$ of pregnancies were either affected by common infections (eg, influenza) or infections manifested as 'fever sub partu' and only rarely resulted in induction of delivery. Other factors that determined preterm birth were isthmocervical insufficiency (20\% of mothers of preterm infants $\leq 32$ weeks GA; $1 \%$ of mothers of term infants), GHT (21\% of mothers of preterm infants; $3 \%$ of mothers of term infants) and placental dysfunction (13\% of mothers of extremely preterm infants; $3 \%$ of mothers of term infants). There was no correlation between GA and self-reported drug or alcohol abuse.

\section{Baseline characteristics of deliveries}

An overview of delivery characteristics is provided in table 4 . In total, $70 \%$ of preterm infants versus $24 \%$ of term infants were delivered via caesarean (C-) section. The largest number of term neonates was born spontaneously (49\%) and only a small proportion was delivered by vacuum extraction or forceps. For all infant groups (extremely preterm, very preterm, late preterm and term), over $60 \%$ of mothers required some form of anaesthesia, mainly delivered via epidural $(40 \%)$ or spinal $(19 \%)$ administration.

\section{Previous pregnancies and deliveries}

Fifty percent of women in the entire parental cohort were primigravida. Mothers of preterm infants had a higher percentage $(13 \%-21 \%)$ of previous preterm deliveries compared with the term group $(3 \%)$.

The percentage of women who had experienced a miscarriage, which refers to pregnancy loss at less than 20 weeks' gestation, was only slightly higher for the preterm (25\%) compared with the term group (19\%). The number of mothers who had lost more than one pregnancy was two times as high $(13 \%)$ for the group of preterm infants $<32$ weeks GA compared with term (6\%, data not shown). Only an exceedingly small percentage of all mothers had induced abortion $(0 \%-6 \%)$ or stillbirth $(0 \%-4 \%)$.

\section{Baseline characteristics of parental study participants}

Anthropometric and demographic characteristics of participating parents are listed in table 5 . The mean age of women from the entire cohort is $34.2 \pm 4.8$ years. Among all mothers, the percentage of women older than 35 years at delivery was higher in all three preterm groups. (54\% for extremely preterm, $52 \%$ for very preterm, $57 \%$ for late preterm) compared with the term group with $45 \%$. Accordingly, the mean age of women who gave birth to extremely preterm infants was higher $(35 \pm 6.1$ years $)$ in comparison to those who delivered term infants (34.1 14.5 years). Due to language barriers, obtaining accurate and extensive self-reported data from non-German and nonEnglish-speaking parents was difficult. Consequently, the MUNICH PreTCl cohort displays limited ethnic parental diversity, with over $90 \%$ of the participating parents reporting a Western European ethnicity. Data on the entire parental cohort portrait a greater proportion of participants who are higher educated than the general population ( $>75 \%$ with university degree) living in urban environments $(75 \%-90 \%)$. 
Table 4 Baseline characteristics of deliveries

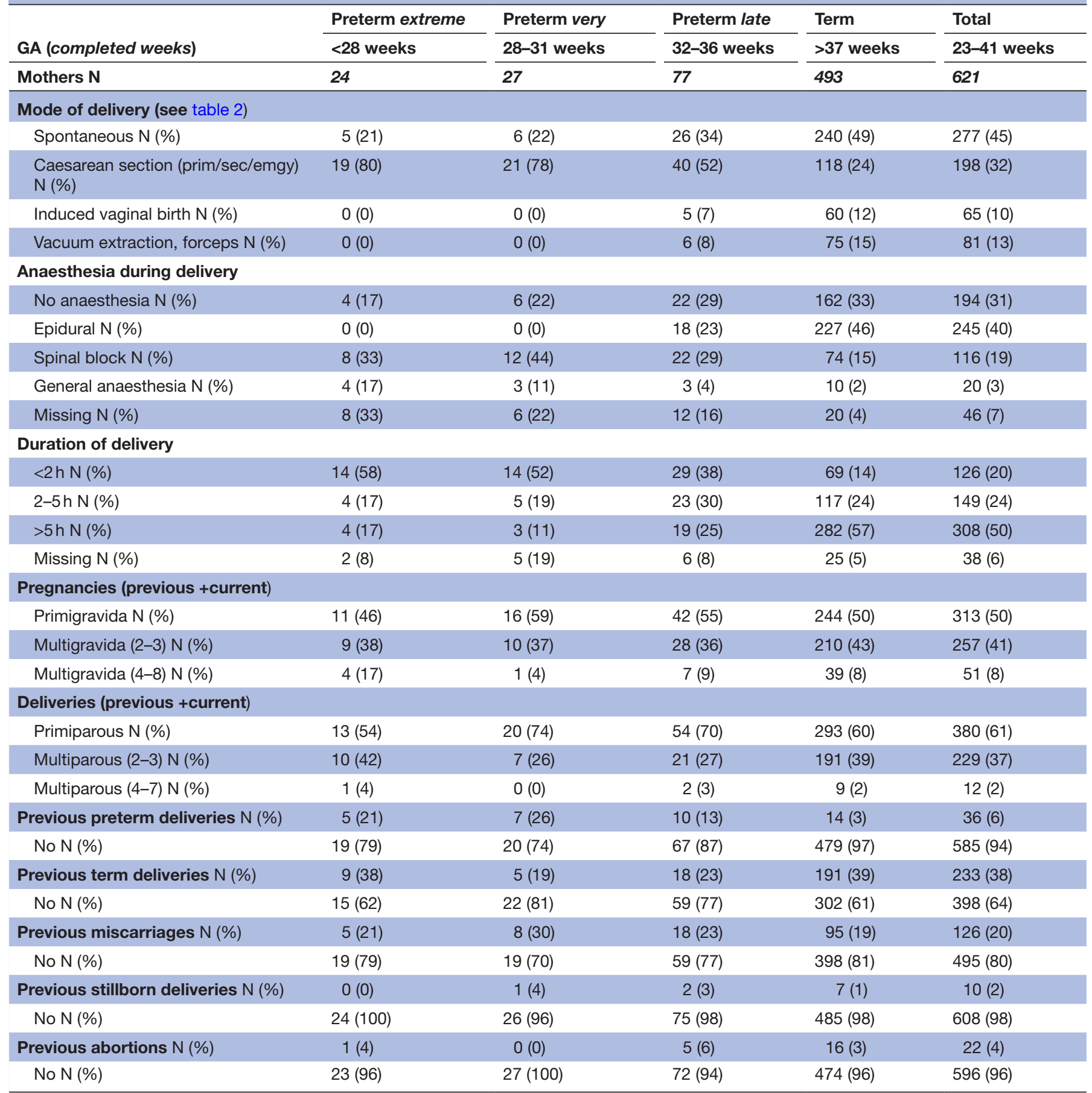

GA, gestational age.

\section{Overview of family medical data}

The family medical history data on parents and their siblings, siblings of enrolled infants and grandparents are shown in table 6 and include information on allergies, asthma, cardiovascular conditions, coagulation disorders, diabetes mellitus as well as neurological and thyroid disorders. A correlation between neonatal or early life infections and allergy or increased risk for asthma has not yet been established in the literature. Previous research suggested that an increased infectious burden in the first
24 month is associated with a decreased prevalence of IgE-mediated allergy during childhood. ${ }^{17}$

In the MUNICH-PreTCl cohort, the percentage of mothers reporting allergies is $43 \%$ and of fathers is $39 \%$. Only about half of these fathers' and mothers' parental generation experienced allergic symptoms. The prevalence of asthma in parents and grandparents was low with 6\%-10\%. Additionally, only a small percentage of mothers and fathers reported cardiovascular diseases, coagulation disorders or neurological abnormalities. There was only a small difference in the 
Table 5 Baseline characteristics of parental study participants

\begin{tabular}{|c|c|c|c|c|c|}
\hline & Preterm extreme & Preterm very & Preterm late & Term & Total \\
\hline GA (completed weeks) & $<28$ weeks & 28-31 weeks & 32-36 weeks & $>37$ weeks & 23-41 weeks \\
\hline Mothers N & 24 & 27 & 77 & 493 & 621 \\
\hline *Survey data: parents $\mathrm{N}$ & 6 & 9 & 51 & 461 & 527 \\
\hline Age: mother (years) M (SD) & $35(6.1)$ & $34.9(5.5)$ & $34.4(5.3)$ & $34.0(4.6)$ & $34.2(4.8)$ \\
\hline$<35$ years N (\%) & $11(46)$ & $13(48)$ & $33(43)$ & $269(55)$ & $326(53)$ \\
\hline$\geq 35$ years $\mathrm{N}(\%)$ & $13(54)$ & $14(52)$ & $44(57)$ & $224(45)$ & $295(47)$ \\
\hline${ }^{*}$ Father (years) M (SD) & $43(6.1)$ & $37.3(4.8)$ & $36.6(5.8)$ & $36(5.8)$ & $36.2(5.9)$ \\
\hline$<35$ years $\mathrm{N}(\%)$ & $1(17)$ & $3(33)$ & $18(35)$ & $187(41)$ & $209(40)$ \\
\hline$\geq 35$ years N (\%) & $5(83)$ & $6(67)$ & $33(65)$ & $274(59)$ & $318(60)$ \\
\hline${ }^{*} \mathrm{BMI}$ : mother (prior pregnancy) M (SD) & $24.5(4.4)$ & $24.2(4.2)$ & $22.8(4.6)$ & $22.4(3.3)$ & $22.5(3.5)$ \\
\hline${ }^{*}$ Father M (SD) & $24.1(1.2)$ & $25.7(2.8)$ & $25.6(3.2)$ & $25.1(3.2)$ & $25.2(3.1)$ \\
\hline \multicolumn{6}{|l|}{ *Adipositas score: mother } \\
\hline Normal N (\%) & $2(33)$ & $6(67)$ & $32(63)$ & $350(76)$ & $390(74)$ \\
\hline Preadipose N (\%) & $1(17)$ & $1(11)$ & $7(14)$ & $68(15)$ & $77(15)$ \\
\hline Adipose N (\%) & $0(0)$ & $1(11)$ & $5(10)$ & $14(3)$ & $20(4)$ \\
\hline Underweight N (\%) & $0(0)$ & $0(0)$ & $3(6)$ & $26(6)$ & $29(5)$ \\
\hline Missing N (\%) & $3(50)$ & $1(11)$ & $4(8)$ & $3(1)$ & $11(2)$ \\
\hline \multicolumn{6}{|l|}{${ }^{*}$ Father } \\
\hline Normal N (\%) & $5(83)$ & $5(56)$ & $25(49)$ & $246(53)$ & $281(53)$ \\
\hline Preadipose N (\%) & $1(17)$ & $4(44)$ & $20(39)$ & $171(37)$ & $196(37)$ \\
\hline Adipose N (\%) & $0(0)$ & $0(0)$ & $5(10)$ & $32(7)$ & $37(7)$ \\
\hline Underweight N (\%) & $0(0)$ & $0(0)$ & $0(0)$ & $1(0.2)$ & $1(0.2)$ \\
\hline Missing N (\%) & $0(0)$ & $0(0)$ & $1(2)$ & $10(3)$ & $10(3)$ \\
\hline \multicolumn{6}{|l|}{${ }^{*}$ Ethnic background: mother } \\
\hline Western N (\%) & $3(50)$ & $7(70)$ & $45(92)$ & $433(94)$ & $488(93)$ \\
\hline Asian N (\%) & $0(0)$ & $1(10)$ & $0(0)$ & 7 (2) & $8(1)$ \\
\hline African and Middle East N (\%) & $3(50)$ & $0(0)$ & $2(4)$ & $15(3)$ & $20(4)$ \\
\hline Latin-American N (\%) & $0(0)$ & $2(20)$ & $1(2)$ & $2(0.4)$ & $5(0.9)$ \\
\hline Indian N (\%) & $0(0)$ & $0(0)$ & $1(2)$ & $4(0.8)$ & $5(0.9)$ \\
\hline \multicolumn{6}{|l|}{ *Father } \\
\hline Western N (\%) & $3(50)$ & $8(89)$ & $45(88)$ & $433(94)$ & $489(93)$ \\
\hline Asian N (\%) & $0(0)$ & $0(0)$ & $1(2)$ & $2(0.4)$ & $3(0.6)$ \\
\hline African and Middle East N (\%) & $3(50)$ & $0(0)$ & 1 (2) & $15(3)$ & $19(4)$ \\
\hline Latin-American N (\%) & $0(0)$ & $1(11)$ & $3(5)$ & $6(1)$ & $10(2)$ \\
\hline Indian N (\%) & $0(0)$ & $0(0)$ & $1(2)$ & $5(1)$ & $6(1)$ \\
\hline \multicolumn{6}{|l|}{ *Education: mother } \\
\hline Certificate $<10$ years school N (\%) & $0(0)$ & $1(11)$ & $1(2)$ & $21(5)$ & $23(4)$ \\
\hline Certificate $\geq 10$ years school $N(\%)$ & $2(33)$ & $4(44)$ & $11(22)$ & $73(15)$ & $90(17)$ \\
\hline University degree $\mathrm{N}(\%)$ & $3(50)$ & $4(44)$ & $37(72)$ & $357(78)$ & $401(76)$ \\
\hline No/other certificate N (\%) & $1(17)$ & $0(0)$ & $2(4)$ & $9(2)$ & $12(2)$ \\
\hline \multicolumn{6}{|l|}{ *Father } \\
\hline Certificate $<10$ years school $\mathrm{N}(\%)$ & $0(0)$ & $1(11)$ & $5(9)$ & $20(4)$ & $26(5)$ \\
\hline Certificate $\geq 10$ years school $N(\%)$ & $0(0)$ & $0(0)$ & $10(19)$ & $76(16)$ & $86(16)$ \\
\hline University degree $\mathrm{N}(\%)$ & $4(80)$ & $8(89)$ & $34(64)$ & $344(75)$ & $390(74)$ \\
\hline No/other certificate N (\%) & $1(20)$ & $0(0)$ & $2(4)$ & $14(3)$ & $17(3)$ \\
\hline Missing N (\%) & $1(20)$ & $0(0)$ & $0(0)$ & 7 (2) & $8(2)$ \\
\hline
\end{tabular}


Table 5 Continued

\begin{tabular}{|c|c|c|c|c|c|}
\hline & Preterm extreme & Preterm very & Preterm late & Term & Total \\
\hline GA (completed weeks) & $<28$ weeks & 28-31 weeks & 32-36 weeks & $>37$ weeks & 23-41 weeks \\
\hline Mothers N & 24 & 27 & 77 & 493 & 621 \\
\hline \multicolumn{6}{|l|}{ *Living environment } \\
\hline Urban N (\%) & $3(50)$ & $7(78)$ & $38(74)$ & $412(89)$ & $460(86)$ \\
\hline Mixed N (\%) & $0(0)$ & $0(0)$ & $4(8)$ & $14(3)$ & $18(4)$ \\
\hline Missing N (\%) & $2(33)$ & $0(0)$ & $1(2)$ & $5(1)$ & $8(2)$ \\
\hline \multicolumn{6}{|l|}{ *Survey } \\
\hline Distributed N & 11 & 13 & 66 & 492 & 582 \\
\hline Completed N & 6 & 10 & 62 & 472 & 550 \\
\hline
\end{tabular}

GA, gestational age.

prevalence of DM (type 1 and type 2, but not GDM) between women and men. The percentage of thyroid disorders was significantly higher in women than in men $(23 \%$ in women and $3 \%$ in men), with hypothyroidism affecting predominantly women (19\% mothers and $0.6 \%$ fathers), probably also due to thyroid screening in pregnancy.

\section{Patient and public involvement}

We regret that we were not aware of patient involvement when we designed and conducted this study. Primarily, our plans did include sharing the study's results with the nurses of the neonatal intensive care unit (NICU) and the newborn ward because they mentioned great interest in our findings. In addition, we will now provide participating families with a copy of this publication to disseminate our study results and provide an opportunity to discuss specific questions on invitation to the 3-year follow-up. For our future research, we will definitely implement active patient contribution.

\section{FINDINGS TO DATE}

To this day no results from the MUNICH-PreTCl study, a birth cohort including preterm and term neonates born over a period of 27 months at the Perinatal Center Campus Innenstadt, University Hospital, LMU, Munich, have been published. The study contains phenotypical information including clinical data from maternal and neonatal medical records, demographics survey and large medical data sets for all families and their neonates as well as reusable dried blood samples obtained at birth and at defined time points throughout hospitalisation, providing the opportunity for further phenotyping using OMICS technologies. These technologies bear great promise to generate extensive and detailed data sets even from very small blood samples and will be an excellent foundation for future systems medicine approaches intended to advance the understanding of complex multifactorial diseases in neonatal and paediatric health.

\section{STRENGTHS AND LIMITATIONS}

The main strength of this study is the combination of a birth cohort with state-of-the-art proteomic screening. This enables us to relate the status of the functional protein network to certain prenatal and postnatal factors as well as specific clinical outcomes that were recorded at time of birth and during follow-up. Our cohort has some limitations. Recruiting at the university hospital and restricting the survey to German and English-speaking parents likely introduced some bias, as mostly caucasian families from urban living environment and with a higher educational level participated in the study. It is not possible to characterise the confounding effect of language barriers to study participation and/or answer accuracy compared with a situation under which the questionnaire would have also been distributed in additional languages, thus being more representative of the 'typical' communitybased population.

The distribution of the questionnaire started with stage 2 of the recruitment process. Consequently, families enrolled in stage 1 (data collection and proteomic analysis method establishment) did not have the opportunity to participate in the survey. Confounding by indication provides another challenge in data analysis. For stage 3 , we enrolled infants with the objective of augmenting specific groups of interest, such as extremely and very preterm infants, neonates with infections, with diabetic mothers or being 'small for GA'. Furthermore, in order to obtain as many data points as possible, we did not exclude families who did not want to participate in the survey.

The interdisciplinary collaboration of experts from various disciplines, such as clinicians, proteomic experts and epidemiologists, will allow a systematic translational approach to find evidence for novel targets that can be applied in clinical practice to improve identification of neonates at risk and advance patient care for a better outcome of preterm infants. 
Table 6 Family medical history

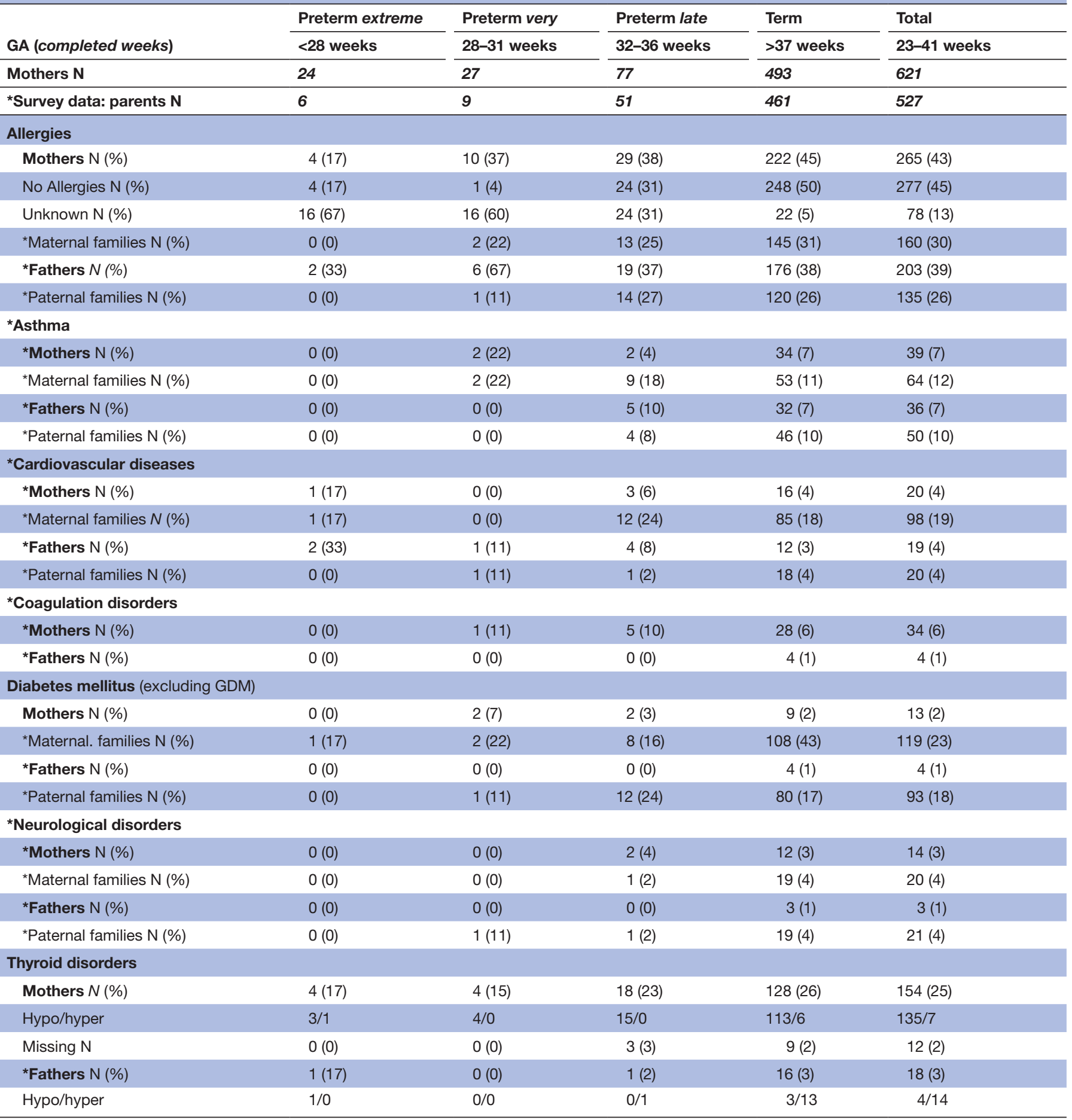

GA, gestational age.

\section{COLLABORATION}

The team of the MUNICH-PreTCl study is very interested in supporting collaborative research on prenatal and postnatal determinants of infant and childhood morbidity and mortality. We would like to enhance possibilities for cooperation by providing a detailed description of the cohort as well as transparency regarding patient recruitment and data collection. Requests for collaborations are welcome and can be made in writing to the corresponding author regarding either data or sample analysis.

\section{FUTURE DIRECTIONS}

Currently, we are in the process of integrating OMIC phenotypes with large clinical data sets, while also coordinating the patients' follow-up visits at the age of 3 years. 
Future plans include data collection of certain subcohorts (eg, infants with family history of allergies) from ages 3-10 years, and in the event of successful funding, this could provide opportunities for additional study visits and even potential biospecimen collection in early childhood.

\section{Author affiliations}

${ }^{1}$ Division of Neonatology Innenstadt, Department of Pediatrics, Dr von Hauner Children's Hospital, University Hospital, LMU, Munich, Germany

${ }^{2}$ Proteomics and Signaltransduction, Max-Planck-Institute of Biochemistry,

Martinsried, Germany

${ }^{3}$ OmicEra Diagnostics GmbH, Planegg, Germany

${ }^{4}$ Nutrition and Epidemiology, Harvard University T H Chan School of Public Health, Boston, Massachusetts, USA

${ }^{5}$ Public Health, University of Copenhagen Faculty of Health and Medical Sciences, Kobenhavn, Denmark

${ }^{6}$ Department of Pediatrics, Dr von Hauner Children's Hospital, University Hospital, LMU, Munich, Germany

${ }^{7}$ Proteomics Program, Novo Nordisk Foundation Centre for Protein Research, University of Copenhagen Faculty of Health and Medical Sciences, Kobenhavn, Denmark

Correction notice This article has been corrected since it was published. Author name corrected to Wolfgang Bodensohn.

Acknowledgements We thank the staff of the NICU and the newborn ward for their excellent and kind cooperation.

Contributors SP-F, PEG, OG-B, CN and MM contributed to the study design. SP-F, $\mathrm{CN}$ and $\mathrm{OG}-\mathrm{B}$ conceived the present analysis and manuscript with significant contributions from MKJ, PEG, JBM-R, JS, WB, RE, NVH, CK and MM. The collection of samples and clinical data were facilitated and coordinated by WB, RE, JS, NVH, AMM. Data management was provided by RE, JS, WB and JBM-R. All authors reviewed and edited the manuscript.

Funding The authors have not declared a specific grant for this research from any funding agency in the public, commercial or not-for-profit sectors.

Competing interests None declared.

Patient and public involvement Patients and/or the public were not involved in the design, or conduct, or reporting, or dissemination plans of this research.

Patient consent for publication Not required.

Ethics approval Data collection and evaluation for this study were approved by the Ethics committee of the medical faculty, LMU Munich, Germany, (Nr 735-15 in 2016) plus Amendments (2017 and 2018).

Provenance and peer review Not commissioned; externally peer reviewed.

Data availability statement The datasets generated and analysed during the current study are available from the corresponding author on reasonable request.

Open access This is an open access article distributed in accordance with the Creative Commons Attribution Non Commercial (CC BY-NC 4.0) license, which permits others to distribute, remix, adapt, build upon this work non-commercially, and license their derivative works on different terms, provided the original work is properly cited, appropriate credit is given, any changes made indicated, and the use is non-commercial. See: http://creativecommons.org/licenses/by-nc/4.0/.
ORCID iDs

Susanne Pangratz-Fuehrer http://orcid.org/0000-0002-5846-9675

Wolfgang Bodensohn http://orcid.org/0000-0002-7062-405X

\section{REFERENCES}

1 Frey HA, Klebanoff MA. The epidemiology, etiology, and costs of preterm birth. Semin Fetal Neonatal Med 2016;21:68-73.

2 Green ES, Arck PC. Pathogenesis of preterm birth: bidirectional inflammation in mother and fetus. Semin Immunopathol 2020;42:413-29.

3 Wilcox AJ, Weinberg CR, Basso O. On the pitfalls of adjusting for gestational age at birth. Am J Epidemiol 2011;174:1062-8.

4 Arbuckle TE, Fraser WD, Fisher M, et al. Cohort profile: the maternalinfant research on environmental chemicals research platform. Paediatr Perinat Epidemiol 2013;27:415-25.

5 Priliani L, Oktavianthi S, Prado EL, et al. Maternal biomarker patterns for metabolism and inflammation in pregnancy are influenced by multiple micronutrient supplementation and associated with child biomarker patterns and nutritional status at 9-12 years of age. PLoS One 2020;15:e0216848.

6 Gaillard R, Welten M, Oddy WH, et al. Associations of maternal prepregnancy body mass index and gestational weight gain with cardio-metabolic risk factors in adolescent offspring: a prospective cohort study. BJOG: Int J Obstet Gy 2016;123:207-16.

7 Bouyssi-Kobar M, du Plessis AJ, McCarter R, et al. Third trimester brain growth in preterm infants compared with in utero healthy fetuses. Pediatrics 2016;138:e20161640.

8 Geyer PE, Holdt LM, Teupser D, et al. Revisiting biomarker discovery by plasma proteomics. Mol Syst Biol 2017;13:942.

9 Blencowe H, Cousens S, Chou D, et al. Born too soon: the global epidemiology of 15 million preterm births. Reprod Health 2013;10:S2.

10 Kramer MS. Determinants of low birth weight: methodological assessment and meta-analysis. Bull World Health Organ 1987;65:663-737.

11 Mendez-Figueroa H, Truong VTT, Pedroza C, et al. Large for gestational age infants and adverse outcomes among uncomplicated pregnancies at term. Am J Perinatol 2017;34:655-62.

12 Poston L, Harthoorn LF, Van Der Beek EM, et al. Obesity in pregnancy: implications for the mother and lifelong health of the child. A consensus statement. Pediatr Res 2011;69:175-80.

13 Rasmussen KM, Catalano PM, Yaktine AL. New guidelines for weight gain during pregnancy: what obstetrician/gynecologists should know. Curr Opin Obstet Gynecol 2009;21:521-6.

14 Dodd JM, Grivell RM, OBrien CM, et al. Prenatal administration of progestogens for preventing spontaneous preterm birth in women with a multiple pregnancy. Cochrane Database Syst Rev 2019;2019. doi:10.1002/14651858.CD012024.pub3. [Epub ahead of print: 20 Nov 2019].

15 Hvidtjørn D, Grove J, Schendel D, et al. Multiplicity and early gestational age contribute to an increased risk of cerebral palsy from assisted conception: a population-based cohort study. Hum Reprod 2010;25:2115-23.

16 Chang H-Y, Hwu W-L, Chen C-H, et al. Children conceived by assisted reproductive technology prone to low birth weight, preterm birth, and birth defects: a cohort review of more than 50,000 live births during 2011-2017 in Taiwan. Front Pediatr 2020;8:87.

17 Nilsson C, Linde A, Montgomery SM, et al. Does early EBV infection protect against IgE sensitization? J Allergy Clin Immunol 2005; $116: 438-44$. 